\title{
Wireless Sensor Networks for Early Detection of Forest Fires
}

\author{
Mohamed Hefeeda and Majid Bagheri \\ School of Computing Science \\ Simon Fraser University \\ Surrey, BC, Canada
}

\begin{abstract}
We present the design and evaluation of a wireless sensor network for early detection of forest fires. We first present the key aspects in modeling forest fires. We do this by analyzing the Fire Weather Index (FWI) System, and show how its different components can be used in designing efficient fire detection systems. The FWI System is one of the most comprehensive forest fire danger rating systems in North America, and it is backed by several decades of forestry research. The analysis of the FWI System could be of interest in its own right to researchers working in the sensor network area and to sensor manufacturers who can optimize the communication and sensing modules of their products to better fit forest fire detection systems. Then, we model the forest fire detection problem as a $k$-coverage problem in wireless sensor networks. In addition, we present a simple data aggregation scheme based on the FWI System. This data aggregation scheme significantly prolongs the network lifetime, because it only delivers the data that is of interest to the application. We validate several aspects of our design using simulation.
\end{abstract}

\section{Introduction}

Forest fires, also known as wild fires, are uncontrolled fires occurring in wild areas and cause significant damage to natural and human resources. Forest fires eradicate forests, burn the infrastructure, and may result in high human death toll near urban areas. Common causes of forest fires include lightning, human carelessness, and exposure of fuel to extreme heat and aridity. It is known that in some cases fires are part of the forest ecosystem and they are important to the life cycle of indigenous habitats. However, in most cases, the damage caused by fires to public safety and natural resources is intolerable and early detection and suppression of fires deem crucial. For example, in August 2003, a forest fire was started by a lightning strike in the Okanagan Mountain Park in the Province of British Columbia, Canada. The fire was spread by the strong wind and within a few days it turned into a firestorm. The fire forced the evacuation of 45,000 residents and burned 239 homes. Most of the trees in the Okanagan Mountain Park were burned, and the park was closed. Although 60 fire departments, 1,400 armed forces troops and 1,000 fire fighters took part in the fire fighting operation, they were largely unsuccessful in stopping the disaster. The official reports estimate the burned area as 25,912 hectares and the total cost as $\$ 33.8$ million [3]. In the Province of British Columbia alone, there have been 2,590 forest fires during 2006 [4]. These burned 131,086 hectares and cost about $\$ 156$ million.

Apart from preventive measures, early detection and suppression of fires is the only way to minimize the damage and casualties. Thus, the most critical issue in a forest fire detection system is immediate response in order to minimize the scale of the disaster. This requires constant surveillance of the forest area. Current medium and large-scale fire surveillance systems do not accomplish timely detection due to low resolution and long period of scan. Therefore, there is a need for a scalable solution that can provide real-time fire detection with high accuracy. We believe that wireless sensor networks can potentially provide such solution. Recent advances in sensor networks support our belief that they make a promising framework for building near realtime forest fire detection systems. Currently, sensing modules can sense a variety of phenomena including temperature, relative humidity, and smoke [7] which are all helpful for fire detection systems [5]. Moreover, recent protocols make sensor nodes capable of organizing themselves into a self-configuring network, thus removing the overhead of manual setup. Large-scale wireless sensor networks can be easily deployed using aeroplanes at a low cost compared to the damages and loss of properties caused by forest fires.

In this paper, we present the design and evaluation of a wireless sensor network for early detection of forest fires. Our design is based on solid forestry research conducted by the Canadian Forest Service [6] over several decades. The rest of the paper is organized as follows. In Sec. 2, we discuss the related work. Sec. 3 describes the FWI System 
which is the basis of our design. The details of our design are presented in Sec. 4. Sec. 5 evaluates various aspects of the proposed system. We conclude the paper in Sec. 6.

\section{Related Work}

Sensor networks have several appealing characteristics for environmental monitoring applications such as habitat monitoring [1,14], and forest fire detection systems $[9,16]$. The authors of [9] show the feasibility of wireless sensor networks for forest fire monitoring. Experimental results are reported from two controlled fires in San Francisco, California. The system is composed of 10 GPS-enabled MICA motes [7] collecting temperature, humidity, and barometric pressure data. The data is communicated to a base station which records it in a database and provides services for different applications. The experiments show that most of the motes in the burned area were capable of reporting the passage of the flame before being burned. In contrast to this system which reports raw weather data, our proposed design processes weather conditions based on the Fire Weather Index System [5] and reports more useful, summarized, fire indexes.

In [10], the authors study the problem of fire behavior rather than fire detection. They present FireWxNet, a portable fire sensor network to measure the weather conditions surrounding active fires. The system is comprised of sensor nodes, webcams, and base stations which are capable of long distance communication. Temperature, relative humidity, wind speed and direction are collected every half an hour while cameras provide a continuous view of the current fire condition. The experimental results indicate that the system is capable of providing useful data for fire behavior analysis. Our system is designed for early detection of forest fires.

A forest fire surveillance system for South Korea mountains using sensor networks is designed in [16]. Sensor nodes use a minimum cost path forwarding to send their readings to a sink which is connected to the Internet. The data is reported to a middleware which calculates a forest fire risk level. Instead of using a middleware, we propose calculating fire indexes according to the FWI System at cell heads where the data is more likely to be correlated. This removes the need for communicating all sensor data to the sink. In our system, only a few aggregated indexes are reported to reduce energy consumption.

\section{Understanding and Modeling Forest Fires}

To assess the possibility of fires starting in forests and rate by which they spread, we adopt one of the most comprehensive forest fire danger rating systems in North Amer-

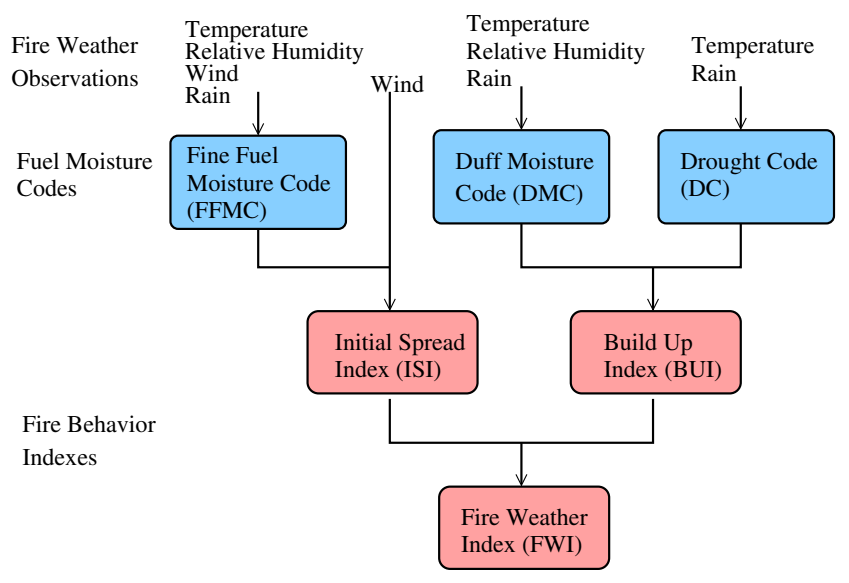

Figure 1. The Fire Weather Index System.

ica. We use the Fire Weather Index (FWI) System developed by the Canadian Forest Service (CFS) [6], which is based on several decades of forestry research [15]. The FWI System estimates the moisture content of three different fuel classes using weather observations. These estimates are then used to generate a set of indicators showing fire ignition potential, fire intensity, and fuel consumption. As shown in Fig. 1, the FWI System is comprised of six components: three fuel codes and three fire indexes. The three fuel codes represent the moisture content of the organic soil layers of forest floor, whereas the three fire indexes describe the behavior of fire. We briefly describe these codes and indexes.

There are three fuel codes in the FWI System: Fine Fuel Moisture Code (FFMC), Duff Moisture Code (DMC), and Drought Code (DC). FFMC represents the moisture content of litter and fine fuels, 1-2 cm deep. Since fires usually start and spread in fine fuels [8], FFMC can be used to indicate ease of ignition, or ignition probability. The Duff Moisture Code (DMC) represents the moisture content of loosely compacted, decomposing organic matter, $5-10 \mathrm{~cm}$ deep. The DMC determines the probability of fire ignition due to lightning and also shows the rate of fuel consumption in moderate depth layers. The last fuel moisture code, the Drought Code (DC), is an indicator of the moisture content of the deep layer of compacted organic matter, $10-20 \mathrm{~cm}$ deep. The DC is indicative of long-term moisture conditions, determines fire's resistance to extinguishing, and indicates fuel consumption in deep layers.

There are three fire indexes: Initial Spread Index (ISI), Buildup Index (BUI), and Fire Weather Index (FWI). As indicated by Fig. 1, ISI and BUI are intermediate indexes and are used to compute the FWI index. The ISI index indicates the rate of fire spread immediately after ignition. It combines FFMC and wind speed to predict the expected rate of fire spread. The BUI index is a weighted combination of 


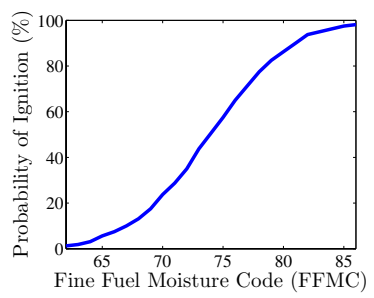

(a) Prob. of ignition vs. FFMC

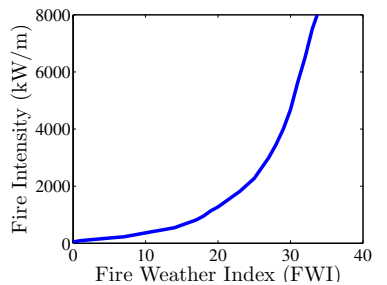

(b) Fire intensity vs. FWI
Figure 2. Using two main components of the FWI System in designing a wireless sensor network to detect forest fires.

the DMC and DC codes, and it indicates the total amount of fuel available for combustion. The FWI index indicates fire intensity by combining the rate of fire spread with the amount of fuel being consumed. Fire intensity is defined as the energy output measured in kilowatts per meter of flame length at the head of a fire. The head of a fire is the portion of a fire edge showing the greatest rate of spread and fire intensity.

There are two goals of the proposed wireless sensor network for forest fires: (i) provide early warning of a potential forest fire, and (ii) estimate the scale and intensity of the fire if it materializes. Both goals are needed to decide on required measures to combat a forest fire. To achieve these goals, we design our sensor network based on the two main components of the FWI System: (i) the Fine Fuel Moisture Code (FFMC), and (ii) the Fire Weather Index (FWI). The FFMC code is used to achieve the first goal and the FWI index is used to achieve the second. In the following, we justify the choice of these two components by collecting and analyzing data from several forestry research publications.

The FFMC indicates the relative ease of ignition and flammability of fine fuels due to exposure to extreme heat. To show this, we interpolate data from [8] to plot the probability of ignition as a function of FFMC. The results are shown in Fig. 2(a). The FFMC scale ranges from 0-101 and is the only component of the FWI System without an openended scale. Generally, fires begin to ignite at FFMC values around 70, and the maximum probable value that will ever be achieved is 96 [8]. Low values of FFMC are not likely to be fires and can simply be ignored, while larger values indicate more alarming situations. The FWI index estimates the fire intensity by combining the rate of fire spread (from the Initial Spread Index, ISI) with the amount of fuel being consumed (from the Buildup Index, BUI). A high value of the FWI index indicates that in case of fire ignition, the fire would be difficult to control. This intuition is backed up by several studies [2,8]. For example, the study in [8] relates the fire intensity with the FWI index. We plot this relationship in Fig. 2(b) by interpolating data from [8].

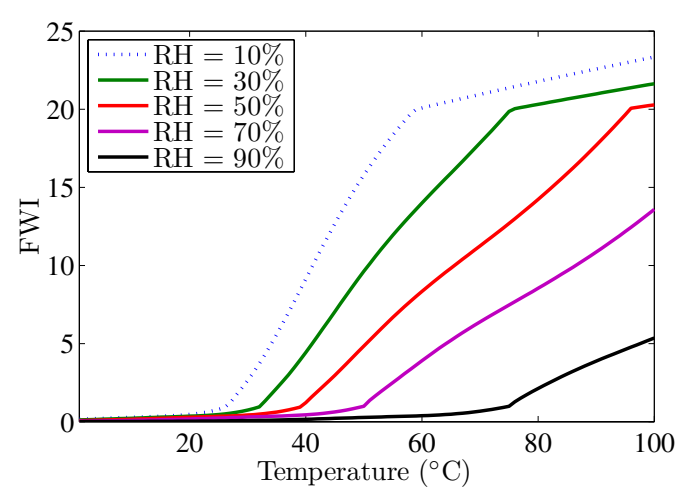

Figure 3. Sensitivity of the FWI Index to temperature.

Both the FFMC code and the FWI index are computed from four basic weather conditions: temperature, relative humidity, precipitation, and wind speed. These weather conditions can be measured by sensors deployed in the forest. The accuracy and the distribution of the sensors impact the accuracy of the FFMC code and the FWI index. Therefore, we need to quantify the impact of these weather conditions on FFMC and FWI. Using this quantification, we can design our wireless sensor network to produce the desired accuracy in FFMC and FWI. To do this, we contacted the Canadian Forest Service to obtain the closed-form equations that describe the dependence of FFMC and FWI on the weather conditions. We were given access to these equations as well as a program that computes them [18]. We used this program to study the sensitivity of FFMC and FWI to air temperature and relative humidity. A sample of our results is presented in Fig. 3, which shows the sensitivity of FWI to temperature for fixed wind speed at $5 \mathrm{~km} / \mathrm{h}$ and precipitation level of $5 \mathrm{~mm}$. We use these results to bound the errors in estimating FFMC and FWI in the next section.

\section{Modeling Forest Fire Detection as a K- Coverage Problem}

In this section, we present the design of a wireless sensor network for forest fire detection. We start by modeling the forest fire detection problem as a $k$-coverage problem. We also extend our distributed $k$-coverage algorithm in [11] to address several issues relevant to forest fire detection. Due to space limitations, we only present the summary of the extensions, more details are given in [12].

We discussed in the previous section the relevance and importance of the FWI System, especially its FFMC and FWI components. We design our wireless sensor network for forest fire detection based on the FWI System. To mon- 
itor a large forest, we need to divide it into smaller subareas. We call each subarea a cell. We periodically compute FFMC and FWI for each cell. This information is then forwarded - through multi hop routing - to a processing center for possible actions. Recall that FFMC and FWI are computed from basic weather conditions such as temperature and humidity (see Fig. 1).

To be useful in detecting fires and assessing their intensity, FFMC and FWI need to be estimated within specific error bounds. For example, if the error in the estimated FWI is high (e.g. 5 units), the fire would be misclassified. To achieve the desired accuracy in FFMC and FWI, basic weather conditions should, in turn, be measured accurately. The accuracy level of measuring basic weather conditions is determined from the curves relating FWI and FFMC to weather conditions. For instance, the worst-case slope of the FWI-Temperature curve in Fig. 3 at $\mathrm{RH}=10 \%$ is about 0.62 . Thus, an error up to 1 unit in FWI requires measuring the temperature with 1.6 degree accuracy. Knowing the needed accuracy in measuring weather conditions, we design our sensor network to collect data with that accuracy. We illustrate this design using temperature as an example, the same can be done for other metrics.

Consider measuring the temperature in an arbitrary cell. We take a number of samples from the cell to estimate the actual temperature. Each sample is collected by a sensor. Multiple samples are needed because of potential errors in readings of sensors. Many factors contribute to such errors, including: different environment conditions (e.g., some sensors happen to be in the shade of trees, while others are not), inaccurate calibration of sensors, aging of sensors, and unequal battery levels in sensors. Let us define a random variable $T$ as the reading of a sensor inside the cell. It is reasonable to assume that $T$ follows a normal distribution because of the several factors mentioned above, which all are stochastic in nature. We denote the mean and standard deviation of $T$ as $\mu_{T}$ and $\sigma_{T}$, respectively. The estimated mean $\widehat{\mu}_{T}$, also known as the sample mean, is given by:

$$
\widehat{\mu}_{T}=\frac{1}{k} \sum_{i=1}^{k} t_{i}
$$

where $t_{i} \mathrm{~s}$ are the individual sensor readings, and $k$ is the number of samples. As the number of samples increases, the sample mean becomes closer to the actual mean. The error between the sample mean and the population mean, $\delta_{T}=\left|\mu_{T}-\widehat{\mu}_{T}\right|$, is calculated as [17]: $\delta_{T}=z_{\frac{\alpha}{2}} \sigma_{T} / \sqrt{k}$, where $z$ is the standard normal distribution, $\alpha$ is the length of the confidence interval, $\sigma_{T}$ is the population standard deviation, and $k$ is the sample size. $z_{\frac{\alpha}{2}}$ can be derived from the table of standard normal distribution. Rearranging the formula, we get:

$$
k=\left(\frac{z_{\frac{\alpha}{2}} \sigma_{T}}{\delta_{T}}\right)^{2} .
$$

Thus, given a confidence value of $100(1-\alpha) \%$ and standard deviation of $\sigma_{T}$, we can determine the sample size required to estimate the population mean $\mu_{T}$ within $\delta_{T}$ error margin. $\sigma_{T}$ can be calculated from the specifications of the sensing board. The error in sensor measurements is usually interpreted as $2 \sigma_{T}$.

Since each temperature sample is collected by a sensor, we need to have at least $k$ active sensors to cover a cell, i.e., the cell is said to be $k$-covered. To illustrate, suppose we want to measure the temperature in a cell with a maximum error of $1^{\circ} \mathrm{C}$ and with a confidence value of $95 \%$. Assume that sensors have temperature sensing boards with an error up to $2^{\circ} \mathrm{C}$, i.e., $\sigma_{T}=1$. Therefore, we need a coverage degree $k=(1.96 \times 1 / 1)^{2}=4$. In the evaluation section, we study and validate the relationship between the coverage degree $k$ and the error in FFMC and FWI.

Keeping $k$ sensors active in a cell all the time will make these sensors die quickly and the sensor network lifetime will be much smaller than even a single fire season (4-6 months). To prolong network lifetime, more sensors need to be deployed and the monitoring task is rotated among them. When sensors are deployed randomly and in high density, the coverage of the cell can be approximated by the coverage of all sensor locations. That is, point coverage is a good approximation for area coverage in dense sensor networks [19]. Now, we need to activate a subset of sensors to ensure $k$-coverage, while other sensors are put in sleep mode to conserve energy. In [11], we present a distributed protocol to achieve this.

Our previous work focused on the theoretical analysis of the algorithm without paying much attention to the specific application. For example, a new issue that was not addressed by the algorithm in [11] is data aggregation and processing. More specifically, we need to compute FFMC and FWI for each cell. To achieve this, we assign each cell a head, which collects weather conditions from other nodes in the cell and computes FFMC and FWI. The assignment of cell heads is done in a distributed manner. Cell heads carry out significant load, because they compute FFMC and FWI from complicated equations and participate in data forwarding across cells. Hence, unless the role of the cell head is rotated, heads run out of energy and die earlier than other nodes. This may cause coverage holes in some areas, or it could partition the network and disrupt data forwarding. More details on the extensions of the distributed $k$-coverage algorithm to address the above and other issues are given in [12], due to space limitations.

\section{Evaluation}

In this section, we evaluate various aspects of the proposed wireless sensor network for forest fire detection. We start by assessing the accuracy in estimating the fire indexes 
FFMC and FWI as a function of the coverage degree $k$. Then, we evaluate the application of our $k$-coverage algorithm to the forest fire detection problem. Due to space limitations, only a sample of the results are presented, more results are given in [12].

We have implemented our own packet-level simulator for the $k$-coverage algorithm in $\mathrm{C}++$. Simulators like NS2 did not scale to the number of nodes (in order of thousands) needed to evaluate our algorithm. We deploy nodes uniformly at random with the same density over the entire area. We fix an area of size $1 \mathrm{~km} \times 1 \mathrm{~km}$ and vary the coverage degree $k$ between 1 and 8 . We set the sensing range of nodes to $100 \mathrm{~m}$. We employ the energy model in [20] and [21], which is based on the Berkeley Mote hardware specifications. In this model, the node power consumption in transmission, reception, idle and sleep modes are 60, 12, 12 , and $0.03 \mathrm{~mW}$, respectively.

We calculate the number of nodes that are initially deployed based on the required network lifetime and the requested coverage degree as follows. The desired network lifetime for a forest fire detection system should be at least one fire season, which is up to six months. Typically, individual nodes cannot last longer than a few weeks. However, since sensors consume energy at a higher rate in active mode compared to sleep mode, a sensor network can last for several months if most nodes are put to sleep. Theoretically, if a node were to be alive throughout the whole network lifetime, it should be active only $p=L_{\text {node }} / L_{\text {network }}$ fraction of the time, where $L_{\text {node }}$ is the node lifetime and $L_{\text {network }}$ is the network lifetime. Knowing $p$, we use the asymptotic sufficient conditions for $k$-coverage proved in [13] to calculate the number of nodes to be deployed. These conditions are obtained for uniformly deployed networks where sensors are active with probability $p$. Since our algorithm activates nodes in a random fashion, we can use these bounds for our initial deployment. Our simulation results presented below validate this assumption. Based on the energy consumption in different modes of current sensor technology (data sheets obtained from Crossbow [7]), we estimated the average sensor lifetime to be around 12 days. Assuming that the desired network lifetime is 6 months, $p$ is 0.067 . Using these values and the conditions in [13], we computed the number of deployed nodes to be $4600,5700,7800,12200$ for $k=1,2,4,8$, respectively.

We summarize our results in the following.

Accuracy of FFMC and FWI. In Section 4, we established a relationship between the coverage degree and the accuracy in estimating FFMC and FWI. We numerically analyze this relationship. We vary the coverage degree $k$ between 1 and 16, and we compute the tolerable error in each case for the weather conditions. We take temperature as example. We fix the confidence level at 95\%. Then, we determine the maximum error in FFMC and FWI from their

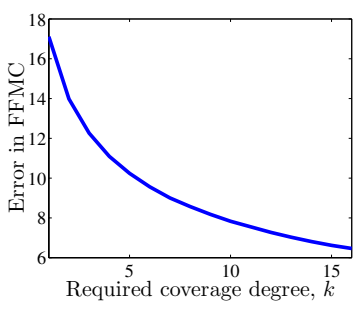

(a)

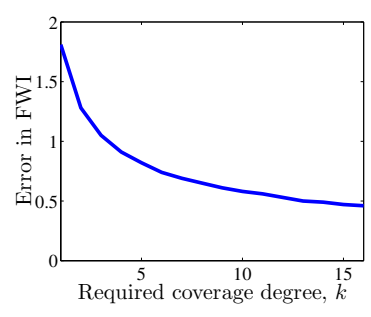

(b)
Figure 4. Error in calculating: (a) FFMC, and (b) FWI for different coverage degrees.

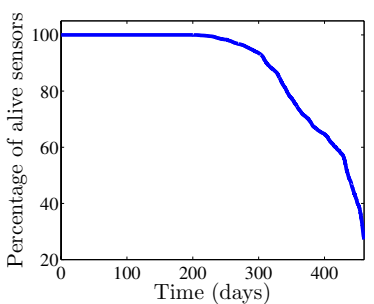

(a) $k=1$

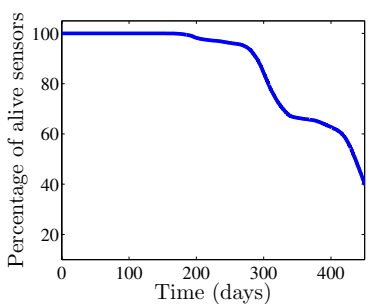

(b) $k=8$

\section{Figure 5. Our $k$-coverage algorithm balances} load across all nodes.

curves. We plot the results in Fig. 4. The figure shows that higher coverage degrees result in smaller errors in FFMC and FWI, as predicted by our analysis in Sec. 4. This figure can be used in choosing the appropriate coverage degree: Given a desired error margin, the coverage degree can be obtained as the maximum of the two values corresponding to FFMC and FWI. For example, if the application can tolerate up to 1 unit in FWI and up to 10 units in FFMC, then a coverage degree of 6 will be sufficient to meet both targets.

Load Balancing. We study the average load on individual nodes and the network lifetime under our $k$-coverage algorithm. We measure the load on a node by the energy consumed by that node. Once a node runs out of energy, it is assumed to be dead. We run our algorithm till all nodes are dead. After each round of the protocol, we count the number of alive nodes. We plot the percentage of alive nodes versus time. We repeat the whole experiment for various coverage degrees, from $k=1$ to $k=8$. Sample of the results are shown in Fig. 5. As the figure shows, most of the nodes stay alive for a long period (more than 200 days). Then, they gradually die. This means that the algorithm did not over utilize some nodes in early rounds, otherwise, they would have died earlier. Notice that the energy of a node is enough for it to be active in a few days, and if a node were chosen as a cell head for several times, it will probably survive for only a few hours. These results confirm that our 
algorithm distributes the load uniformly across all deployed nodes. This is critical in order to keep nodes alive for the longest possible period and achieve more reliable coverage. This also extends the network lifetime.

\section{Conclusions}

We presented the design of a wireless sensor network for early forest fire detection. Our design is based on the Fire Weather Index (FWI) System. We showed how the forest fire detection problem can be modeled as a $k$-coverage problem. We computed the required coverage degrees to achieve a given accuracy level in estimating different components of the FWI System. We then presented a distributed $k$-coverage algorithm to solve the $k$-coverage problem. Our algorithm is simple to implement and does not require any specific node deployment schemes. Therefore, nodes can be uniformly deployed by, for example, throwing them from an aircraft. This significantly facilitates node deployment in real life. We also presented a data aggregation scheme based on the FWI System. We showed through simulations that our algorithm balances load across all deployed nodes, and therefore maintains reliable coverage and significantly prolongs the network lifetime.

\section{Acknowledgment}

This work is partially supported by the Natural Sciences and Engineering Research Council (NSERC) of Canada under Discovery Grant \#313083 and RTI Grant \#344619.

\section{References}

[1] I. Akyildiz, S. Weilian, Y. Sankarasubramaniam, and E. Cayirci. A survey on sensor networks. IEEE Coтmunications Magazine, 40(8):102-114, August 2002.

[2] M. Alexander and W. D. Groot. Fire behavior in Jack Pine stands as related to the Canadian Forest Fire Weather Index System. Technical report, Canadian Forest Service, Northern Forestry Centre, Edmonton, Alberta, 1988.

[3] B.C. Ministry of Forests and Range. Fire Review Summary for Okanagan Mountain Fire (K50628).

[4] B.C. Ministry of Forests and Range Web Page. http://www.for.gov.bc.ca.

[5] Canadian Forest Fire Danger Rating System (CFFDRS) Web Page. http://www.nofc.forestry.ca/fire.

[6] Canadian Forest Service (CFS) Web Page. http://www.nrcan.gc.ca/cfs.

[7] Crossbow Inc. Web Page. http://www.xbow.com/.

[8] W. J. de Groot. Interpreting the Canadian Forest Fire Weather Index (FWI) System. In Proc. of the Fourth Central Region Fire Weather Committee Scientific and Technical Seminar, Edmonton, Canada, 1998.
[9] D. M. Doolin and N. Sitar. Wireless sensors for wildfire monitoring. In Proc. of SPIE Symposium on Smart Structures and Materials, pages 477-484, San Diego, CA, March 2005.

[10] C. Hartung, R. Han, C. Seielstad, and S. Holbrook. FireWxNet: A multi-tiered portable wireless system for monitoring weather conditions in wildland fire environments. In Proc. of International Conference on Mobile systems, Applications and Services (MobiSys'06), pages 28-41, Uppsala, Sweden, June 2006.

[11] M. Hefeeda and M. Bagheri. Randomized k-coverage algorithms for dense sensor networks. In Proc. of IEEE INFOCOM 2007 Minisymposium, pages 2376-2380, Anchorage, AK, May 2007.

[12] M. Hefeeda and M. Bagheri. Wireless sensor networks for early detection of forest fires. Technical Report TR 200708, School of Computing Science, Simon Fraser University, Surrey, BC, Canada, April 2007.

[13] S. Kumar, T. H. Lai, and J. Balogh. On k-coverage in a mostly sleeping sensor network. In Proc. of ACM International Conference on Mobile Computing and Networking (MOBICOM'04), pages 144-158, Philadelphia, PA, September 2004.

[14] A. Mainwaring, D. Culler, J. Polastre, R. Szewczyk, and J. Anderson. Wireless sensor networks for habitat monitoring. In Proc. of the First International Workshop on Wireless Sensor Networks and Applications (WSNA'02), pages 88-97, Atlanta, Georgia, September 2002.

[15] J. San-Miguel-Ayanz, J. Carlson, M. Alexander, K. Tolhurst, G. Morgan, and R. Sneeuwjagt. Chapter 2: Current methods to assess fire danger potential. In Wildland Fire Danger Estimation and Mapping - The Role of Remote Sensing Data. World Scientific Publishing Co. Pte. Ltd., 2003.

[16] B. Son, Y. Her, and J. Kim. A design and implementation of forest-fires surveillance system based on wireless sensor networks for South Korea mountains. International Journal of Computer Science and Network Security (IJCSNS), 6(9):124-130, 2006.

[17] J. R. Taylor. Introduction to Error Analysis. University Science Books, second edition, 1997.

[18] C. V. Wagner and T. Pickett. Equations and FORTRAN program for the Canadian Forest Fire Weather Index System. Technical Report 33, Canadian Forest Service, Ottawa, Ontario, 1985.

[19] S. Yang, F. Dai, M. Cardei, and J. Wu. On connected multiple point coverage in wireless sensor networks. International Journal of Wireless Information Networks, 13(4):289-301, May 2006.

[20] F. Ye, G. Zhong, S. Lu, and L. Zhang. PEAS: A robust energy conserving protocol for long-lived sensor networks. In Proc. of the 23rd International Conference on Distributed Computing Systems (ICDCS'03), pages 28-37, Providence, RI, May 2003.

[21] H. Zhang and J. Hou. Maintaining sensing coverage and connectivity in large sensor networks. Ad Hoc and Sensor Wireless Networks: An International Journal, 1(1-2):89_ 123, January 2005. 